\title{
Jogo para Mensuração de Habilidades Preditoras de Leitura: Construção e Análise
}

\author{
Jucelio Soares dos Santos¹, Monilly Ramos Araújo de Melo, Dalton Dario Serey \\ Guerrero $^{1}$, Jorge César Abrantes Figueiredo ${ }^{1}$, Carla Alexandra Silva Moita \\ Minervino $^{2}$
}

${ }^{1}$ Universidade Federal de Campina Grande

2 Universidade Federal da Paraíba

jucelio@copin.ufcg.edu.br, monillyramos@gmail.com, \{dalton, abrantes\}@dsc.ufcg.edu.br, carlamoitaminervino@gmail.com

\begin{abstract}
The objective of this paper is to present a description of the development of a digital game in order to improve the screening capacity of children in the identification of dyslexia signs in assessments of the cognitive abilities predictive of reading development. The game is an adaptation of the "Teste de Habilidades Preditoras da Leitura", a psychometric instrument built and validated in Brazil. The game is administered by an adaptive selection of items and designed to measure the skills of alliteration, segmentation, visual memory and rhyme by of Item Response Theory. As a result, the paper highlights the importance of the use of game elements associated to computerized adaptive assessment and its effective applicability conditions.
\end{abstract}

Resumo - O objetivo deste trabalho é apresentar uma descrição do desenvolvimento de um jogo digital, com intuito de melhorar a capacidade de triagem de crianças, identificando sinais de dislexia em avaliações das habilidades cognitivas preditoras do desenvolvimento da leitura. O jogo é uma adaptação do "Teste de Habilidades Preditoras da Leitura", um instrumento psicométrico construído e validado no Brasil. O jogo é administrado por um algoritmo de seleção adaptativa de itens e projetado para mensurar as habilidades de aliteração, segmentação, memória visual e rima por meio da Teoria de Resposta ao Item. Como resultado, o trabalho evidencia a importância do uso de elementos de jogos associados a avaliação adaptativa informatizada e as suas condições efetivas na aplicabilidade.

\section{Introdução}

Em nossa realidade educacional tornou-se comum o encaminhamento de crianças aos Centros de Atendimento Educacional Especializado (CAEE) com suspeitas e até mesmo laudos médicos atestando quadros de dislexia ${ }^{1}$ e outros distúrbios de aprendizagem ${ }^{2}$. Porém, por intermédio de um processo de triagem no próprio CAEE, nota-se que um grande número de alunos apresenta limitações na leitura geradas por fatores ambientais

\footnotetext{
${ }^{1}$ Déficit no processamento fonológico responsável pela dificuldade na decodificação e relação entre letras e sons [Catts \& Kahmi, 1999].

2 Envolvem situações orgânicas, ou seja, o indivíduo já nasce com o distúrbio que o dificulta em seu aprendizado [Carvalho, Crenitte \& Ciasca, 2007].
} 
VII Congresso Brasileiro de Informática na Educação (CBIE 2018)

Anais do XXIX Simpósio Brasileiro de Informática na Educação (SBIE 2018)

ou metodológicos, sobrecarregando o sistema público de ensino com a síndrome do encaminhamento ${ }^{3}$.

Por exemplo, na cidade de Campina Grande, Paraíba, Brasil, constatou-se que crianças são encaminhadas erroneamente ao único centro responsável pelo diagnóstico no município - Fundação Papel Marchê. Em seu relatório anual, em 2016, apenas 12,6\% das crianças encaminhadas foram diagnosticadas com dislexia. Entre as que não foram diagnosticadas como disléxicas, verificou-se a existência de outros problemas como: 3,6\% com Transtorno do Espectro Autista, 8,4\% com Deficiência Intelectual e 75,4\% com Dificuldade de Aprendizagem [Fundação Papel Marchê, 2016].

Tal problema é ocasionado principalmente pela formação acadêmica dos educadores que não se sentem familiarizados sobre os processos cognitivos e neurofuncionais relacionados à leitura. Outrossim, as propostas pedagógicas que prevalecem no sistema educacional brasileiro não evidenciam, durante a fase de alfabetização, a relação grafema fonema, uma das habilidades preditoras da aquisição proficiente da leitura. Além disso, as escolas não possuem mecanismos pedagógicos adequados para o rastreamento dos fatores de risco da dislexia, dificultando o processo de identificação de crianças com limitações na leitura [Capellini, 2004]. Esta realidade poderia ser melhorada se os profissionais da educação dispusessem de um recurso que pudesse auxiliá-los a detectar limitações na leitura em crianças, orientando-os na elaboração de métodos interventivos para adequadamente facilitar o encaminhamento e, consequentemente, diminuir o número de crianças encaminhadas com limitações ocasionadas por dificuldades de aprendizagem, que poderiam ser abordadas nas escolas com uma metodologia de ensino adequada [Dias and Melo, 2015].

Um caminho que já existe para solucionar tal problemática se refere à utilização de testes psicométricos baseados na Teoria de Resposta ao Item (TRI), administrados por um algoritmo adaptativo informatizado que seleciona de forma adequada os itens em relação ao nível de habilidade do sujeito. A TRI considera o item como unidade básica de análise e procura representar a probabilidade de um indivíduo dar uma resposta ao item em função dos seus parâmetros e da sua habilidade. Tais bancos de itens podem auxiliar profissionais na área a resolverem problemas que requerem ferramentas de qualidade além do conhecimento especializado. Neles são aplicados o mesmo conjunto de itens para todos os indivíduos, possibilitando maior precisão, celeridade, facilidade de atualização, além de que, os testes são menos sujeitos a erros na divulgação dos seus resultados [Sartes and Souza-Formigoni, 2013]. Entretanto, apesar desses avanços, a criança pode se sentir avaliada nos testes estáticos e fornecer respostas não confiáveis [Joly and Reppold, 2010].

Visando preservar o estado pleno do bem-estar físico e mental da criança, partiuse da ideia de que elementos de jogos podem encorajar comportamentos específicos e motivar os participantes durante a avaliação, consistindo na aplicação de elementos lúdicos em atividades comuns para torná-las mais atrativas, divertidas e eficazes. Dessa forma, o Laboratório de Práticas de Software (SPLAB), o Laboratório de Neuropsicologia Cognitiva e Inovação Tecnológica (LabNEUROCIT), o Núcleo de Estudos de Saúde Mental, Educação e Psicometria (NESMEP) e a Fundação Papel Marchê vêm

\footnotetext{
${ }^{3}$ Transferência de responsabilidade dos professores à órgãos responsáveis por atendimento educacional especializado, espera-se um diagnóstico que não é condizente com a realidade educacional do aluno [Vasconcellos, 1994].
} 
VII Congresso Brasileiro de Informática na Educação (CBIE 2018)

Anais do XXIX Simpósio Brasileiro de Informática na Educação (SBIE 2018)

desenvolvendo essa pesquisa concentrada na utilização de jogos administrados por algoritmos adaptativos que avaliam as habilidades preditoras de leitura em crianças por meio da TRI.

Na dislexia, os jogos educacionais são fortes aliados para estimular a criação de habilidades na leitura, reforçando a aprendizagem de forma estruturada e multissensorial. Por meio dos jogos é possível que a criança revise constantemente um assunto, proporcionando um trabalho individual seguindo o ritmo do aluno. Além disso, é possível que o indivíduo faça o uso de diferentes atividades, estimulando o desenvolvimento das habilidades cognitivas e emocionais [Santos et al, 2014].

Mediante esses aspectos, é possível explorar técnicas modernas de mensuração da habilidade nas atividades dos jogos. Essa representação é um avanço para as áreas de avaliação neurocognitiva e educacional, por abordar conceitos da psicometria moderna que mensura a habilidade baseada nas respostas fornecidas pelo usuário, visando assim rastrear as limitações das crianças [Pasquali, 2004]. Entretanto, na literatura e no mercado existem poucas ferramentas construídas com este delineamento e para esse propósito, algumas ainda em fase de pesquisa [Soares, Munzlinger and Riveros, 2005] [Zapirain, Zorilla, and Bartolome, 2012]. Poucos são os instrumentos computadorizados confiáveis, cuja validade foi cientificamente comprovada, que podem ser utilizados para o rastreamento de casos que devem ser encaminhados a um especialista.

Entre os recursos tecnológicos adaptativos disponíveis para levantamento de indícios de dislexia, destaca-se o Teste de Habilidades Preditoras de Leitura (THPL) [Moita, 2013], que mensura as habilidades de aliteração, segmentação, memória visual e rima dos participantes por meio da TRI. Retomando um dos aspectos da problemática exposta anteriormente, no que diz respeito ao engajamento do examinando na tarefa de avaliação, levantou-se a hipótese de que sua aplicação em meios tradicionais pode inibir a criança, comprometendo os resultados obtidos. Uma alternativa foi encapsular o THPL em abordagem de jogo, de forma a trazer à criança um instrumento lúdico que gerasse maior empatia.

Para investigar essa questão foi desenvolvido um jogo intitulado "Os Niridianos no Inacreditável Mar de Letras” adaptado do THPL e administrado por um algoritmo de seleção adaptativa de itens, que mensura as habilidades preditoras de leitura por meio da TRI. O objetivo do jogo é instrumentalizar os professores, por meio da aplicação das tarefas de rastreio, para a identificação de crianças com riscos de apresentarem dislexia. A construção e análise do jogo é apresentada neste artigo.

\section{Construção do Jogo}

O jogo “Os Niridianos no Inacreditável Mar de Letras” foi desenvolvido a partir das tarefas de consciência fonológica (aliteração, segmentação e rima) e memória visual do THPL [Moita, 2013]. Além destas tarefas presentes no teste, o jogo também consegue explorar, devido a sua abordagem, à tarefa de coordenação viso-motora.

Na tarefa aliteração a criança é avaliada com base na repetição de uma sequência de sons de palavras que contém o mesmo início, sendo pedido que a mesma selecione, dentro de três opções, qual a palavra que contém o mesmo início da indicada, conforme a Figura 1. A tarefa segmentação avalia a criança na identificação do número de sílabas da palavra em tela. Nesta atividade é pedido a criança que selecione, dentro de cinco 
VII Congresso Brasileiro de Informática na Educação (CBIE 2018)

Anais do XXIX Simpósio Brasileiro de Informática na Educação (SBIE 2018)

opções, aquele referente a quantidade de sílabas que a palavra proposta possui. A tarefa memória visual, por sua vez, avalia a capacidade de processamento de imagens. Esta tarefa se configura na observação de uma figura em um curto intervalo de tempo e, em seguida, na sua identificação diante de um conjunto maior de desenhos. A tarefa rima avalia a criança no que diz respeito à repetição de uma sequência de sons de palavras que contém a mesma terminação. Nesta tarefa é proposta uma escolha, dentre três opções, a fim de identificar a palavra que contém a mesma terminação daquela indicada.

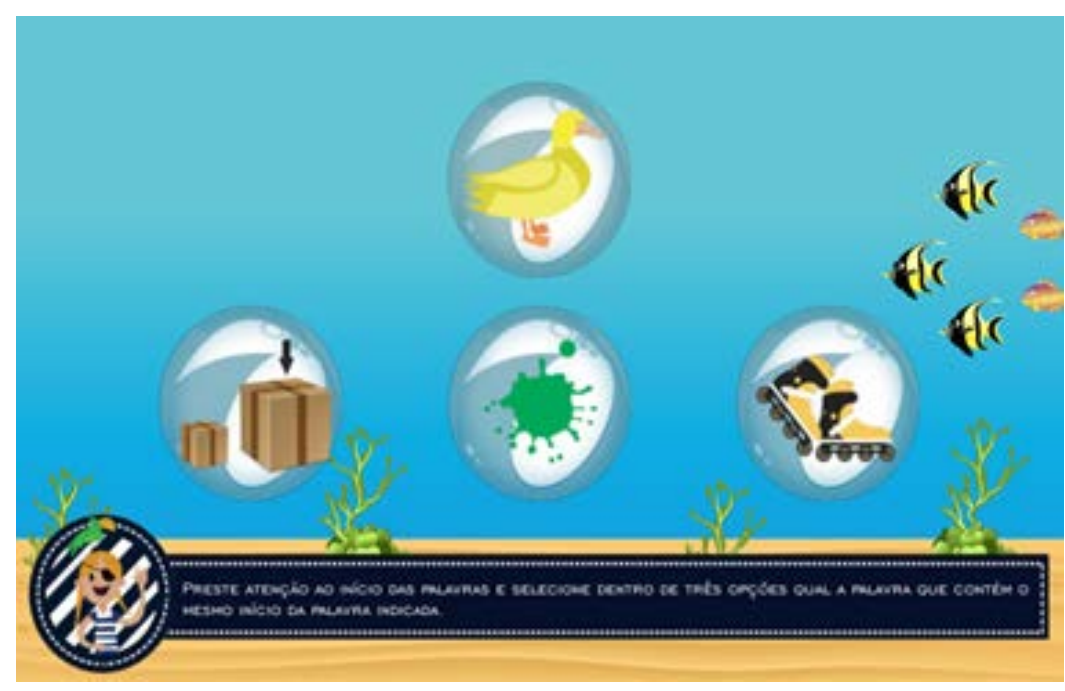

Figura 1. Exemplo da Tarefa Aliteração

Por fim, tem-se a tarefa coordenação viso-motora, que avalia a criança no que tange ao direcionamento intencional dos olhos, controlando a acuidade visual, ou seja, a capacidade de ver e diferenciar objetos apresentados no campo visual com significado e precisão. É pedido a criança que recupere o tesouro perdido no menor tempo possível e colidindo com as cavernas o mínimo de vezes, conforme a Figura 2.

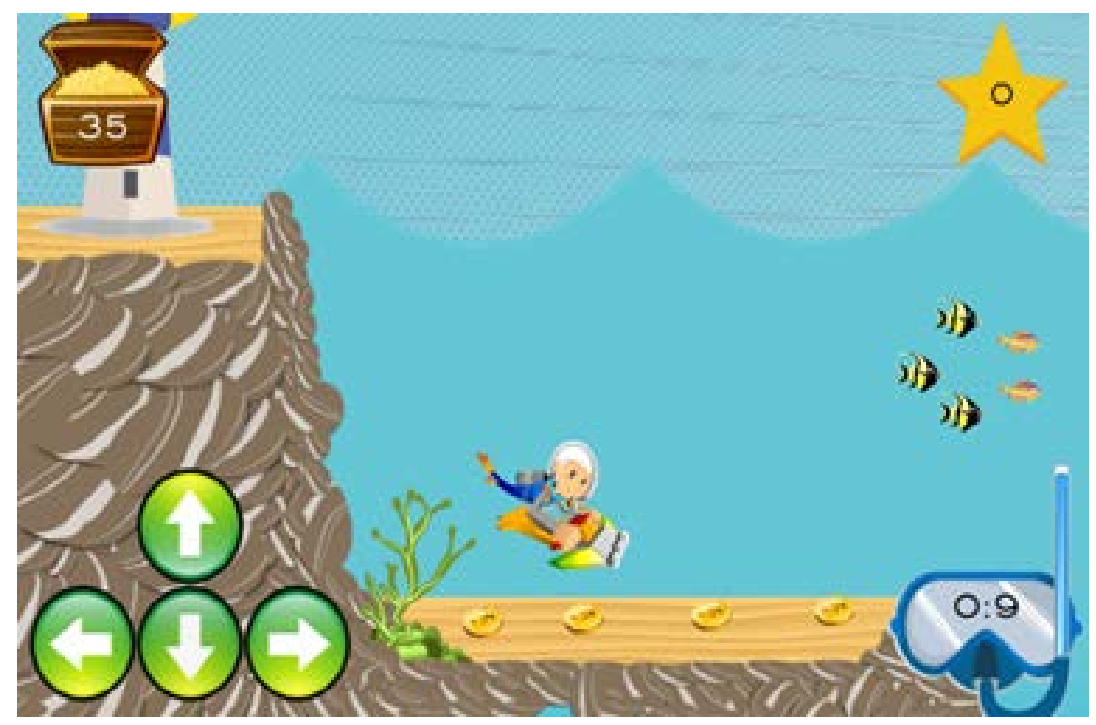

Figura 2. Exemplo da Tarefa Coordenação Viso-Motora

No processo de construção do jogo foram necessários dois elementos básicos: i) banco de itens com questões de boa qualidade do ponto de vista pedagógico e psicométrico; e, ii) um algoritmo para seleção adaptativa dos itens. 
VII Congresso Brasileiro de Informática na Educação (CBIE 2018)

Anais do XXIX Simpósio Brasileiro de Informática na Educação (SBIE 2018)

\subsection{Banco de Itens}

Para o banco de itens, foram consideradas as seguintes etapas: i) elaboração dos itens mediante a um conjunto de palavras selecionadas do teste com os respectivos desenhos gráficos e arquivos de áudio; e, ii) a calibração dos itens para suas propriedades psicométricas.

Este estudo é parte de um estudo maior, intitulado Avaliação Adaptativa Informatizada para Levantar Indícios de Dislexia em Crianças na Fase de Alfabetização, cuja realização foi aprovada pelo Comitê de Ética em Pesquisa com seres humanos da Universidade Federal de Campina Grande (UFCG) (Protocolo: 55160816.6.0000.5182). Apenas as crianças cujos pais assinaram o Termo de Assentimento participaram da pesquisa.

\subsection{Elaboração dos Itens}

A partir de uma lista contendo 2.957 palavras de baixa, média e alta frequência de ocorrência no vocabulário de leitura das crianças de pré-escola e $1^{\circ}$ a $5^{\circ}$ ano do ensino fundamental, selecionamos 170 palavras que pudessem ser representadas através de uma imagem e que estivessem presentes nas listas de cada ano escolar [Pinheiros, 1996]. Devese salientar que o critério de escolha das palavras foi pré-definido pelo THPL [Moita, 2013] e autorizado pelo NESMEP e LabNEUROCIT apenas para uso restrito de pesquisa. Através da biblioteca gratuita freepik (http://br.freepik.com/), foi baixada para cada palavra uma ilustração vetorizada, sendo que algumas foram modificadas por meio de uma ferramenta de edição de gráficos, a CorelDraw Graphics Suíte X8. Para efetivação dos desenhos, levou-se em consideração a idade de 4 a 13 anos das crianças. Ao todo foram selecionados/modificados 170 desenhos para compor os itens. Após a aprovação do projeto no Comitê de Ética em Pesquisa da UFCG, os participantes e os responsáveis pelas crianças assinaram o Termo de Consentimento Livre e Esclarecido (TCLE) anuindo a sua participação no estudo que se iniciou logo após a fase conclusão dos desenhos, quando os mesmos foram apresentados a três grupos amostrais com intuito de analisar e identificar os desenhos por parte dos grupos, verificando, para tanto, o grau de concordância entre os respondentes. Os desenhos que obtiveram $80 \%$ de concordância por parte dos participantes de cada grupo foram selecionados para a construção dos itens das tarefas de aliteração, segmentação, memória visual e rima, os que não atingiram o nível pré-determinado de concordância foram descartados.

Participaram deste estudo: i) um grupo de juízes composto por 3 (três) professores do ensino fundamental e 2 (dois) professores universitários do Centro de Educação da UFCG, sendo 1 (um) homem e 4 (quatro) mulheres, com idade superior a 30 anos e nível superior de escolaridade; ii) um grupo de 25 (vinte e cinco) crianças oriundas de escolas públicas municipais de Campina Grande, Paraíba, Brasil. A faixa etária variou entre 6 a 12 anos (idade média = 8 anos e 6 meses; $\mathrm{dp}=1,32$ ), sendo $28 \%$ do sexo feminino e $72 \%$ do sexo masculino; iii) e um terceiro grupo composto de 26 (vinte e seis) universitários do Centro de Educação da UFCG, sendo todos do sexo feminino com idade entre 19 e 28 anos.

Após a análise das respostas de cada grupo de avaliadores, foram selecionados 147 desenhos. Vinte e três desenhos foram excluídos por não atenderem ao pré-requisito de $80 \%$ de concordância entre os respondentes. Uma vez selecionadas as figuras, foram 
VII Congresso Brasileiro de Informática na Educação (CBIE 2018)

Anais do XXIX Simpósio Brasileiro de Informática na Educação (SBIE 2018)

utilizados os itens presentes no THPL, bem como os seus áudios, que foram gravados por uma jornalista sem sotaque regional. Os itens foram escolhidos de forma a abarcar itens fáceis, médios e difíceis de serem resolvidos, divididos, portanto, em 3 níveis. Para as tarefas de aliteração, segmentação, memória visual e rima foram selecionados 120 itens. Para estipular o grau de dificuldade foram consideradas a extensão, frequência, tonicidade e regularidade da palavra-estímulo [Moita, 2013].

\subsection{Calibração dos Itens}

Após a escolha dos itens do jogo, foram verificadas as propriedades psicométricas dos itens. Esse procedimento é uma etapa importante na construção de qualquer instrumento, pois possibilita verificar se a escala construída se encontra minimamente adequada para que seja dada continuidade ao estudo. Os itens foram apresentados na própria execução de um protótipo do jogo em tablets com mesma configuração. O jogo foi aplicado no laboratório de informática de cada escola em grupos de 6 crianças, para aquelas que foram consentidas pelos pais. As respostas das crianças durante a aplicação do jogo foram transformadas em itens do tipo certo/errado (itens dicotômicos), foi atribuído 0 ao errar e 1 ao acertar.

Participaram deste estudo: 270 crianças, sendo 134 (49,63\%) meninas e 136 meninos (50,37\%), com idade entre 4 e 13 anos (idade média =8,65; dp =2,08), alunos de escolas públicas municipais de Campina Grande, Paraíba, Brasil. Considerou-se como critérios de inclusão: a frequência na escola e estar matriculado nos anos iniciais do ensino fundamental I. Excluíram-se todas as crianças que apresentaram comprometimentos motores, cognitivos ou da comunicação que pudessem prejudicar os resultados. Esta informação foi fornecida pelas educadoras de cada uma das salas de aula. Vale destacar que o processo de escolha da amostra foi caracterizado como disponível, tendo-se estimado somente as crianças que, no momento da aplicação do jogo, estavam presentes na sala de aula.

Os dados coletados nesta fase do estudo foram analisados pela TRI com o auxílio da ferramenta R-Studio por meio dos pacotes R que você pode usar para análise e ajuste do modelo logístico de três parâmetros (ML3) com a finalidade de verificar: i) a consistência interna do instrumento (correlação entre diferentes itens no mesmo teste) e, ii) a estimativa dos parâmetros dos itens das tarefas do jogo.

A consistência interna do instrumento pode ser calculada por meio do pacote ltm com a função cronbach.alpha( ), a saída pode ser vista na Tabela 1.

Tabela 1. Consistência interna do instrumento

\begin{tabular}{|c|c|c|c|}
\hline Tarefa & Média & Desvio Padrão & Cronbach’s Alpha \\
\hline Aliteração & 23,922 & 6,563 & 0,921 \\
\hline Segmentação & 17,659 & 7,634 & 0,913 \\
\hline Memória Visual & 26,426 & 5,435 & 0,925 \\
\hline Rima & 21,533 & 7,134 & 0,913 \\
\hline
\end{tabular}


VII Congresso Brasileiro de Informática na Educação (CBIE 2018)

Anais do XXIX Simpósio Brasileiro de Informática na Educação (SBIE 2018)

A partir dos valores obtidos para o alfa de Cronbach, conclui-se que os resultados obtidos na avaliação do instrumento são confiáveis para todas atividades apresentadas.

A interpretação da distribuição das respostas das crianças em cada item das tarefas foi feita a partir dos parâmetros do ML3, além disso foram consideradas a proporção de acertos e a correlação ponto bisserial entre a resposta correta no item e a pontuação total na tarefa. Os parâmetros podem ser calculados por meio do pacote ltm com a função tpm() e por meio do mesmo pacote é possível calcular a correlação ponto bisserial dos itens com a função biserial.cor(), uma parte da saída pode ser vista na Tabela 2, em que são apresentados os primeiros 5 itens dos 30 que compõem a tarefa aliteração com os respectivos parâmetros, os quais foram considerados como conhecidos neste trabalho. A tabela completa dos itens e de todas as tarefas está disponível em https://goo.gl/n3e81U.

Tabela 2. Tarefa Aliteração, 5 primeiros itens calibrados

\begin{tabular}{|c|c|c|c|c|c|c|}
\hline Id & Item & a & b & c & p.a. & c.p.b. \\
\hline 001 & Pato & 1,768 & $-1,545$ & 0,142 & 0,881 & 0,497 \\
\hline 002 & Carro & 1,714 & $-0,83$ & 0,13 & 0,759 & 0,536 \\
\hline 003 & Bota & 1,903 & $-1,536$ & 0,154 & 0,889 & 0,495 \\
\hline 004 & Preto & 2,821 & $-0,876$ & 0,216 & 0,826 & 0,566 \\
\hline 005 & Folha & 1,923 & $-0,752$ & 0,214 & 0,781 & 0,489 \\
\hline \multicolumn{2}{|c|}{ Média } & 2,032 & $-0,946$ & 0,169 & 0,797 & 0,512 \\
\hline \multicolumn{2}{|c|}{ Desvio padrão } & 0,496 & 0,350 & 0,031 & 0,064 & 0,081 \\
\hline
\end{tabular}

Dado a: parâmetro de discriminação do item; b: parâmetro de dificuldade do item; c: parâmetro de probabilidade de acerto ao acaso; p.a.: probabilidade de acerto; e, c.p.b.: correlação ponto bisserial.

Com intuito de evitar o comprometimento da representatividade do domínio do avaliado, foi verificado se os itens atendiam às condições exigidas dos seus parâmetros em relação ao ML3. Não foram encontrados valores críticos para os parâmetros estimados, todos os itens das tarefas do jogo possuem valores superiores a 0,30 para o índice de discriminação; para o índice de dificuldade valores entre 2,95 e -2,95; e, a probabilidade de acerto casual abaixo de 0,40. Em relação a probabilidade de acerto, os resultados revelaram que as tarefas aliteração e rima apresentam itens fáceis (com índices acima de $75 \%$ ) e itens moderados (com índices entre 50 a 75\%). A tarefa segmentação apresenta itens moderados e itens difíceis (com índices abaixo de 50\%). E, a tarefa memória visual apresenta apenas itens fáceis. A correlação ponto-bisserial revelou uma tendência de escolha da opção errada pelas crianças que obtiveram escores mais altos no teste. Por exemplo, entre os 5 primeiros itens da tarefa aliteração, o item 005 é o que menos influencia no escore total dos alunos que obtiveram bom desempenho no teste.

Após perceber que não houve necessidade de excluir itens nas atividades, deu-se início o próximo passo, a construção do algoritmo para seleção adaptativa dos itens das tarefas do jogo.

\subsection{Algoritmo Utilizado}


VII Congresso Brasileiro de Informática na Educação (CBIE 2018)

Anais do XXIX Simpósio Brasileiro de Informática na Educação (SBIE 2018)

O jogo é gerenciado segundo um algoritmo de seleção. Esse algoritmo especifica os itens que a criança irá responder e sua ordem de apresentação. $\mathrm{O}$ algoritmo é composto por 3 (três) partes: i) como começar? Qual o primeiro item a ser respondido? Devido à falta de informações prévias, será escolhido um item aleatório a um nível de habilidade entre o intervalo $(-1,1)$. Utilizamos a Máxima Informação de Fisher para selecionar o primeiro item, nesse caso, o item que possui mais informação; ii) como continuar? Depois de cada resposta, qual o próximo item? Após cada nova resposta, atualizamos a estimativa da habilidade pela Máxima Verossimilhança e a usamos para selecionar o próximo item; iii) quando parar? Quando o teste termina? É indispensável determinar o momento em que o indivíduo não responderá mais itens (critério de parada). Optou-se por utilizar uma combinação de três fatores: número mínimo e máximo de itens aplicados conjugado com o valor mínimo para o erro padrão encontrado no cálculo da habilidade. Depois, foi elaborado um query para encontrar o último item a ser aplicado por tarefa caso o critério de parada fosse a variação do erro padrão no cálculo da habilidade, com valor menor que 0,01. Estes valores foram encontrados mediante um script que simulou a aplicação do teste com base nas respostas dadas pelas crianças no pré-teste. Desta forma, foram encontrados os seguintes resultados para o critério de parada por tarefa, conforme a Tabela 3.

Tabela 3. Número mínimo e máximo de itens nas Tarefas do jogo

\begin{tabular}{|l|c|c|c|c|}
\hline \multicolumn{1}{|c|}{ Tarefa } & Média & Variância & Min. & Max. \\
\hline Aliteração & 8,304 & 17,914 & 4,835 & 11,213 \\
\hline Segmentação & 9,593 & 16,509 & 4,531 & 12,370 \\
\hline Memória Visual & 6,267 & 14,463 & 4,899 & 8,054 \\
\hline Rima & 8,589 & 19,876 & 4,655 & 11,782 \\
\hline
\end{tabular}

Por exemplo, na tarefa Aliteração, o algoritmo não selecionará mais itens quando no mínimo for administrado 5 (cinco) itens e/ou o erro padrão no cálculo da habilidade for menor que 0,01 ou no máximo 11 (onze) itens.

\section{Análise do Jogo}

Com intuito de verificar o desempenho do instrumento desenvolvido, foram dimensionados dois parâmetros a serem observados: a precisão na estimativa interina da habilidade e o seu tempo de aplicação. Para a precisão, foi estimada a habilidade das crianças no pré-teste pela Expectativa a Posteriori (EAP) através do pacote irtoys e a função $\operatorname{eap}($ ) e a correlacionamos com a habilidade gerada pelo instrumento reduzido (com critério de parada) e o instrumento completo (administrando todos os itens do instrumento).

Como os dados não seguem uma distribuição normal, foi utilizado o método de Spearman para verificar a correlação entre a habilidade estimada pela EAP e as demais habilidades estimadas pelo instrumento reduzido e completo. Desta forma, foram encontrados os seguintes resultados da correlação por tarefa (Tabela 4). 
VII Congresso Brasileiro de Informática na Educação (CBIE 2018)

Anais do XXIX Simpósio Brasileiro de Informática na Educação (SBIE 2018)

Tabela 4. Correlação entre a habilidade estimada pela EAP e a habilidade estimada do instrumento reduzido/completo em cada tarefa

\begin{tabular}{|l|c|c|}
\hline \multicolumn{1}{|c|}{ Tarefa } & Instrumento reduzido & Instrumento completo \\
\hline Aliteração & 0,837 & 0,746 \\
\hline Segmentação & 0,856 & 0,690 \\
\hline Memória Visual & 0,690 & 0,645 \\
\hline Rima & 0,851 & 0,726 \\
\hline
\end{tabular}

De acordo com a Tabela 4 existe uma correlação forte entre a habilidade estimada pela EAP e as habilidades estimadas pelo teste reduzido/completo para as tarefas de aliteração e rima. Na tarefa de memória visual esta correlação é moderada para os dois casos. Já na tarefa de segmentação, essa correlação é forte e moderada, respectivamente. E, em todas as tarefas do jogo, a correlação é maior para a habilidade estimada pelo teste que possui o critério de parada.

Uma das vantagens do uso de um algoritmo para seleção adaptativa dos itens é a economia do tempo. Se fosse utilizado o teste completo, ou seja, sem critério de parada, em média a criança demoraria cerca de 42 minutos para completar o teste. Já no teste reduzido, este tempo foi otimizado. Na simulação, as crianças levaram em média 13 minutos para completar o instrumento. Como resultado, a automação do instrumento permitirá a economia do tempo, facilidade de reprodução, bem como a obtenção de resultados mais precisos da habilidade dos participantes.

\section{Considerações Finais}

Partindo dos achados disponíveis na literatura acerca das contribuições da Tecnologia da Informação para os processos de avaliação e intervenção na saúde e educação, implementou-se, no presente estudo, a construção de um instrumento de avaliação das habilidades preditoras de leitura com abordagem de jogo. Em virtude da escassez de instrumentos com essa abordagem e, visando, um delineamento metodológico que considera evidências científicas de validade já publicadas na área, partiu-se de uma ferramenta já construída e validada para a mensuração das mesmas habilidades cognitivas, o THPL [Moita, 2013].

Assim, no presente artigo, foi apresentado o procedimento de elaboração do jogo “Os Niridianos no Inacreditável Mar de Letras”, dividido em várias etapas, a saber: Elaboração dos itens, na qual foi relatada toda a metodologia e as técnicas utilizadas no desenvolvimento dos itens que compõem o jogo; Calibração dos Itens e construção da escala, na qual foi relatada a condução do estudo e a análise das propriedades psicométricas dos itens; e, por último, a Elaboração do algoritmo, etapa em que se detalhou o processo de construção do algoritmo com base no critério de partida, no método estatístico para estimar o traço latente, no procedimento para selecionar o próximo item e no critério de parada.

É importante mencionar que a hipótese de vantagem do jogo sobre o THPL não foi mensurada neste estudo, porque seria necessário obter suas primeiras evidências de validade psicométrica para que se possa utilizá-lo, posteriormente, de maneira 
VII Congresso Brasileiro de Informática na Educação (CBIE 2018)

Anais do XXIX Simpósio Brasileiro de Informática na Educação (SBIE 2018)

equivalente ao THPL, equivalente em termos psicométricos. Assim, propõe-se, na próxima etapa da pesquisa, a aplicação de uma escala de usabilidade para a criança e para o profissional com objetivo de verificar o seu nível de satisfação e engajamento nas duas modalidades.

\section{Referências}

Capellini, S. A. (2004). Distúrbios de aprendizagem versus dislexia. In L. Ferreira, D. Befi-Lopes, \& S. Limongi (Eds.), Tratado de fonoaudiologia (pp. 862-876). São Paulo: Roca.

Carvalho, F. B., Crenitte, P. A. P., \& Ciasca, S. M. (2007). Distúrbios de aprendizagem na visão do professor. Revista Psicopedagogia, 24(75), 229-239.

Catts, H. W., \& Kahmi, A. G. (1999). Language and reading disabilities (1st ed.). Boston: Allyn Bacon.

Dias, C. S., Melo, M. R. A. (2015). Avaliação psicopedagógica com crianças e adolescentes com queixas de dificuldade de aprendizagem. In Melo, M. R. A. (Org.), Desenvolver: Um programa de avaliação e intervenção neuropsicológica para crianças e adolescentes com dificuldades de aprendizagem (pp. 35-52). Curitiba: CRV.

Fundação Papel Machê (2016). Relatório Anual. Campina Grande: Fundação Papel Machê.

Joly, M. C. R. A. \& Reppold, C. T. (2010). Testes informatizados para a avaliação psicológica e educacional. E-book. São Paulo: Casa do Psicólogo.

Moita, P. M. S. (2013). Avaliação adaptativa em dispositivos móveis das habilidades cognitivas preditoras do desenvolvimento de leitura em crianças (Unpublished master's thesis). Universidade de Lisboa. Instituto de Educação. Faculdade de Ciências. Programa de Pós-Graduação em Tecnologias e Metodologias em E-learning, Lisboa.

Pasquali, L. (2004). Psicometria: Teoria dos testes na Psicologia e na Educação. (2st ed.). Petrópolis: Editora Vozes.

Pinheiro, A. M. (1996). Contagem de Frequência de Ocorrência de Palavras Expostas a Crianças na Faixa Pré-escolar e Séries Iniciais do $1^{\circ}$ Grau (1st ed.)., São Paulo: Associação Brasileira de Dislexia.

Sartes, L. M., \& Souza-Formigoni, M. L. (2013). Avanços na psicometria: da teoria clássica dos testes à teoria de resposta ao item. Psicologia: Reflexão e Crítica, 26(2), 241-250.

Santos, J. S. Costa, R. A., Souza, R. P., Pereira, I. B. \& Pereira, R. S. O. (2014). Proposta de um jogo educacional para alfabetização de crianças com dislexia. Workshop de Informática na Escola, (20):457-466.

Soares, F. S. Munzlinger, E. \& Riveros, L. J. M. (2005). Validação de um sistema especialista para o pré-diagnóstico da dislexia. Congresso da Sociedade Brasileira de Computação, (25).

Vasconcellos, C. d. S. (1994). Disciplina: Construção da disciplina consciente e interativa em sala de aula e na escola (1st ed.). São Paulo: Libertad.

Zapirain, B. G. Zorilla, A. M. \& Bartolome., N. A. (2012). Dyslexia diagnosis in reading stage though the use of games at school. International Conference on Computer Games, (17):12-17. 